\title{
EQUATIONS OF MOTION OF SPINNING RELATIVISTIC PARTICLE IN EXTERNAL FIELDS
}

\author{
I.B. Khriplovich and A.A. Pomeransky \\ Budker Institute of Nuclear Physics, 630090 Novosibirsk, Russia \\ and Novosibirsk University \\ E-mail: khriplovich@inp.nsk.su,pomeransky@vxinpz.inp.nsk.su
}

\begin{abstract}
We consider the motion of a spinning relativistic particle in external electromagnetic and gravitational fields, to first order in the external field, but to an arbitrary order in spin. The noncovariant spin formalism is crucial for the correct description of the influence of the spin on the particle trajectory. We show that the true coordinate of a relativistic spinning particle is its naive, common coordinate $\vec{r}$. Concrete calculations are performed up to second order in spin included. A simple derivation is presented for the gravitational spinorbit and spin-spin interactions of a relativistic particle. We discuss the gravimagnetic moment (GM), a specific spin effect in general relativity. It is shown that for the Kerr black hole the gravimagnetic ratio, i.e., the coefficient at the GM, equals unity (just as for the charged Kerr hole the gyromagnetic ratio equals two). The equations of motion obtained for relativistic spinning particle in external gravitational field differ essentially from the Papapetrou equations.
\end{abstract}

\footnotetext{
${ }^{1}$ Plenary talk at the Fairbank Meeting on the Lense-Thirring Effect, Rome-Pescara, 29/6-4/7 1998
} 


\section{Introduction}

The problem of the motion of a particle with internal angular momentum (spin) in an external field consists of two parts: the description of the spin precession and accounting for the spin influence on the trajectory of motion. To lowest nonvanishing order in $c^{-2}$ the complete solution for the case of an external electromagnetic field was given more than 70 years ago [1]. The gyroscope precession in a centrally symmetric gravitational field had been considered to the same approximation even earlier [2]. Then, much later the spin precession was investigated in the case of the gravitational spin-spin interaction [3]. The fully relativistic problem of the spin precession in an external electromagnetic field was also solved more than 70 years ago [四, and then in a more convenient formalism, using the covariant vector of spin, in [5].

The situation with the second part of the problem, which refers to how the spin influences the trajectory, is different. Covariant equations of motion for a relativistic spinning particle in an electromagnetic field were written in the same paper [4], and for the case of a gravitational field in [6]. These equations have been discussed repeatedly from various points of view in numerous papers (see, e.g., [7-18]). The problem of the influence of the spin on the trajectory of a particle in external fields is not only of purely theoretical interest. It attracts attention being related to the description of the motion of ultrarelativistic particles in accelerators [19] (see also recent review [20]).

In fact, it is far from being obvious whether one can observe in practice the discussed spin corrections to the equations of motion of elementary particles, say, electron or proton. According to the well-known argument by Bohr (see [21]), an additional Lorentz force due to the finite size of the wave packet of a charged particle and to the uncertainty relation, exceeds the corresponding component of the Stern-Gerlach force. However, this argument by itself does not exclude in principle the possibility to observe a regular Stern-Gerlach effect, let even a small one, in the presence of a larger background due to the uncertainty relation. This possibility is supported, in particular, by recent calculations [22]. Moreover, spin-dependent correlations certainly exist in differential cross sections of scattering processes. So, it was proposed long ago to separate by polarizations a beam of charged particles in a storage ring through the spin interaction with external fields [23]. Though this proposal is being discussed rather actively (see review 20]), it is not 
clear up to now whether it is feasible technically.

There are however macroscopic objects for which internal rotation certainly influences their trajectories. We mean the motion of Kerr black holes in external gravitational fields. This problem is of importance in particular for the calculation of the gravitational radiation of binary stars. In this connection it was considered in [24-27]. However, when we turned to these calculations, we found [28] that the equations of motion with spin taken into account to the lowest nonvanishing order in $c^{-2}$, used in these papers, even in the simpler case of an external field lead to results which differ from the well-known gravitational spin-orbit interaction. The problem is essentially related to the correct definition of the center-of-mass coordinate. Moreover, it turned out that the widely used Papapetrou equations [6] also fail to reproduce in the same $c^{-2}$ approximation the result for the gravitational spin-orbit interaction found in the classical work [2]. This discrepancy was pointed out long ago in 229; however the explanation suggested in 29 does not look satisfactory (see [28]).

In our recent work [30 (the present talk is essentially based on it) we derived equations of motion of a relativistic particle with the noncovariant description of spin. These equations agree with well-known limiting cases. Though for external electromagnetic field such equations in the linear in spin approximation have been obtained previously [19] (see also [20]), we would like to start with comments related to this approximation in electrodynamics.

\section{Covariant and Noncovariant Equations of Motion of a Spinning Particle in an Electromagnetic Field}

\subsection{The Problem with Covariant Equations of Motion}

The interaction of spin with external electromagnetic field is described, up to terms on the order of $c^{-2}$ included, by the well-known Hamiltonian (see, for example, [31])

$$
H=-\frac{e g}{2 m} \vec{s} \vec{B}+\frac{e(g-1)}{2 m^{2}} \vec{s}[\vec{p} \times \vec{E}]
$$


Here $\vec{B} \quad \vec{E}$ are external magnetic and electric fields; $e, m, \vec{s}$, and $\vec{p}$ are the particle charge, mass, spin, and momentum, respectively; $g$ is its gyromagnetic ratio. Let us emphasize that the structure of the second (Thomas) term in this expression not only has been firmly established theoretically, but has also been confirmed with high accuracy experimentally, at any rate in atomic physics. To avoid misunderstandings, let us note that, generally speaking, the last term in formula (1) should be rewritten in a Hermitian form (see, e.g., [32):

$$
[\vec{p} \times \vec{E}] \rightarrow \frac{1}{2}([\vec{p} \times \vec{E}]-[\vec{E} \times \vec{p}])=[\vec{p} \times \vec{E}]+\frac{i}{2} \nabla \times \vec{E} .
$$

We will be interested, however, in the semiclassical approximation mainly, when in the interaction linear in spin, field derivatives are neglected. (Besides, the correction with $\nabla \times \vec{E}$ vanishes in the case of potential electric field considered in [31].)

Let us try to construct a covariant equation of motion accounting for spin, which would reproduce in the same approximation the force

$$
\vec{f}_{m}=\frac{e g}{2 m} \vec{s} \vec{B},_{m}+\frac{e(g-1)}{2 m}\left(\frac{d}{d t}[\vec{E} \times \vec{s}]_{m}-\vec{s}\left[\vec{v} \times \vec{E},_{m}\right]\right),
$$

corresponding to the Hamiltonian (四) (here and below coma with a subscript denotes a partial derivative). A covariant correction $f^{\mu}$ to the Lorentz force $e F^{\mu \nu} u_{\nu}$, linear in the tensor of spin $S_{\mu \nu}$ and in the gradient of the tensor of electromagnetic field $F_{\mu \nu, \lambda}$, may depend also on the 4 -velocity $u^{\mu}$. Since $u^{\mu} u_{\mu}=1$, this correction must satisfy the condition $u_{\mu} f^{\mu}=0$. From the mentioned tensors one can construct only two independent structures meeting the last condition. The first,

$$
\eta^{\mu \kappa} F_{\nu \lambda, \kappa} S^{\nu \lambda}-F_{\lambda \nu, \kappa} u^{\kappa} S^{\lambda \nu} u^{\mu}
$$

reduces in the $c^{-2}$ approximation to

$$
2 \vec{s}\left(\vec{B},,_{m}-\left[\vec{v} \times \vec{E}_{, m}\right]\right),
$$

and the second,

$$
u^{\lambda} F_{\lambda \nu, \kappa} u^{\kappa} S^{\nu \mu}
$$


reduces to

$$
\frac{d}{d t}[\vec{s} \times \vec{E}]_{m}
$$

(Let us note that the structures with the contraction $F_{\nu \kappa, \lambda} S^{\kappa \lambda}$ reduce to these two expressions, due to the Maxwell equations and the antisymmetry of $S_{\kappa \lambda}$.)

Obviously, no linear combination of these two structures can reproduce the correct expression (2) for the spin-dependent force. In a somewhat less general way it was shown in 28.

But why the correct (in the $c^{-2}$ approximation) formula (2) cannot be obtained from a covariant expression for the force? Obviously, one can easily reproduce those terms in (2) which are proportional to $g$ by a linear combination of (3) and (4), i.e., there is no problem to present in a covariant form the terms which describe, so to say, direct interaction of a magnetic moment with external fields. It is the terms in (2) independent of $g$ and corresponding to the Thomas precession which cannot be written covariantly. Certainly, the Thomas precession can be described beyond the $c^{-2}$ approximation, for arbitrary velocities. But there are no reasons why this essentially noncovariant phenomenon should have a covariant description. This is the point.

\subsection{What Is the Correct Definition of the Coordinate of a Spinning Particle?}

It was noted in [28] that the covariant formalism can be reconciled with the correct results if the coordinate $\vec{x}$ entering the covariant equation is related to the usual one $\vec{r}$ in the $c^{-2}$ approximation as follows:

$$
\vec{x}=\vec{r}+\frac{1}{2 m} \vec{s} \times \vec{v} .
$$

- The generalization of this substitution to the case of arbitrary velocities

$$
\vec{x}=\vec{r}+\frac{\gamma}{m(\gamma+1)} \vec{s} \times \vec{v}, \quad \gamma=\frac{1}{\sqrt{1-v^{2}}} .
$$

was recently pointed out in 20.

But why the spin precession itself (as distinct from the spin influence on the trajectory) can be described covarianly [ [4, 5] without any concern for 
the coordinate definition? First of all, the covariant equations of the spin precession

$$
\frac{d S_{\mu}}{d \tau}=\frac{e}{2 m}\left[g F_{\mu \nu} S^{\nu}-(g-2) u_{\mu} F_{\lambda \nu} u^{\lambda} S^{\nu}\right]
$$

(here $S_{\mu}$ is the covariant 4 -vector of spin) are written in the semiclassical approximation, i.e., the coordinate dependence of external fields is neglected at all. Second, equations (17) are homogeneous and linear in spin. So, even if one went here beyond the semiclassical approach, but stayed within the approximation linear in spin, the use of the usual coordinate $\vec{r}$, which differs from $\vec{x}$ in terms proportional to $\vec{s}$ only, would be completely legitimate.

Of course, the choice of the variable, $\vec{r}$ or $\vec{x}$, is by itself a matter of convention. But still, which of them is the true center-of-mass coordinate of a relativistic spinning body?

We note first of all that relation (5) is valid for a free particle as well. So, to answer the question, we will consider a simple example of the free Dirac particle with the Hamiltonian

$$
H=\vec{\alpha} \vec{p}+\beta m \text {. }
$$

Here, the operator whose expectation value equals to $\vec{r}$, is not $\vec{r}$ itself, but [33]

$$
\vec{x}=\vec{r}+\frac{i \beta \vec{\alpha}}{2 \epsilon}-\frac{i \beta(\vec{\alpha} \vec{p}) \vec{p}+[\vec{\Sigma} \times \vec{p}] \epsilon}{2 \epsilon^{2}(\epsilon+m)} ; \quad \epsilon=\sqrt{p^{2}+m^{2}} ; \quad \vec{\Sigma}=\frac{1}{2 i}[\vec{\alpha} \times \vec{\alpha}] .
$$

To lowest nonvanishing order in $c^{-2}$ expression (\$) reduces to

$$
\vec{x}=\vec{r}+\frac{1}{2 m} \vec{s} \times \vec{v}, \quad \vec{s}=\frac{1}{2} \vec{\sigma},
$$

which might prompt indeed substitution (5). However, under the FoldyWouthuysen (FW) transformation, which separates positive-energy states from negative-energy ones, the relativistic operator $\vec{x}$ goes over into mere $\vec{r}$. And the transition from the exact Dirac equation in an external field to its approximate form containing only the first-order correction in $c^{-2}$ is performed just by means of the FW transformation. Thus, in the arising $c^{-2}$ Hamiltonian the coordinate of a spinning electron is the same $\vec{r}$ as in the completely nonrelativistic case. Nobody makes substitution (5) in the Coulomb potential when treating the spin-orbit interaction in the hydrogen atom. 
As to a classical particle, it is in fact a well-localized wave packet constructed from positive-energy states, i.e., it is properly described in the FW representation. Therefore, it is $\vec{r}$ which is the true coordinate of a classical relativistic spinning particle.

\subsection{The Noncovariant Formalism}

The correct equations of motion in an electromagnetic field including spin to first order are known for a fairly long time [19]. Though being fully relativistic, they are noncovariant and based on the initial physical definition of spin. According to this definition, spin is the 3-dimensional vector $\vec{s}$ (or 3-dimensional antisymmetric tensor $s_{m n}$ ) of the internal angular momentum defined in the rest frame of the particle. The covariant vector of spin $S_{\mu}$ (or the covariant antisymmetric tensor $S_{\mu \nu}$ ) are obtained from $\vec{s}$ (or $s_{m n}$ ) merely by the Lorentz transformation. By the way, an advantage of this approach is that the constraints $u^{\mu} S_{\mu}=0$ and $u^{\mu} S_{\mu \nu}=0$ hold identically. The precession frequency for spin $\vec{s}$ at an arbitrary velocity is well-known (see, for instance, [31]):

$$
\begin{aligned}
\vec{\Omega}=\frac{e}{2 m}\{ & (g-2)\left[\vec{B}-\frac{\gamma}{\gamma+1} \vec{v}(\vec{v} \vec{B})-\vec{v} \times \vec{E}\right] \\
& \left.+2\left[\frac{1}{\gamma} \vec{B}-\frac{1}{\gamma+1} \vec{v} \times \vec{E}\right]\right\} .
\end{aligned}
$$

Naturally, the corresponding interaction Lagrangian (the Lagrangian description is here somewhat more convenient than the Hamiltonian one) equals

$$
\begin{aligned}
L_{1 s}=\vec{\Omega} \vec{s}=\frac{e}{2 m} \vec{s} & \left\{(g-2)\left[\vec{B}-\frac{\gamma}{\gamma+1} \vec{v}(\vec{v} \vec{B})-\vec{v} \times \vec{E}\right]\right. \\
+ & {\left.\left[\frac{1}{\gamma} \vec{B}-\frac{1}{\gamma+1} \vec{v} \times \vec{E}\right]\right\} . }
\end{aligned}
$$

The equation of motion for coordinate is the usual one:

$$
\left(\nabla-\frac{d}{d t} \nabla_{\vec{v}}\right) L_{t o t}=0
$$


where $L_{t o t}$ is the total Lagrangian of the system. The equation of motion for spin in general form is

$$
\dot{\vec{s}}=-\left\{L_{t o t}, \vec{s}\right\},
$$

where $\{\ldots, \ldots\}$ is the Poisson bracket, or

$$
\dot{\vec{s}}=-i\left[L_{t o t}, \vec{s}\right]
$$

in the quantum problem. This applicability of a common canonical formalism is one more advantage of the noncovariant approach. Meanwhile, in the covariant approach we have to deal in particular with higher time derivatives, which is obvious already from relation (9).

\section{Equations of Motion of a Spinning Particle in an Electromagnetic Field}

\subsection{The General Approach}

Our approach to the problem is based on the following physically obvious argument. As long as we do not consider excitations of internal degrees of freedom of a body moving in an external field, this body (even if it is macroscopic) can be treated as an elementary particle with spin.

Therefore, the Lagrangian of the spin interaction with an external field can be derived from the elastic scattering amplitude

$$
-e J^{\mu} A_{\mu}
$$

of a particle with spin $s$ by a vector potential $A_{\mu}$. Due to the arguments presented in Section 1, the discussion of the effects nonlinear in spin (which are of primary interest to us) may be physically meaningful only in the classical limit $s \gg 1$. It is this approximation that is basically used below.

The matrix element $J_{\mu}$ of the electromagnetic current operator between states with momenta $k$ and $k^{\prime}$ can be written (under $P$ and $T$ invariance) as follows (see, for instance, 34, 35]):

$$
J_{\mu}=\frac{1}{2 \epsilon} \bar{\psi}\left(k^{\prime}\right)\left\{p_{\mu} F_{e}+\Sigma_{\mu \nu} q^{\nu} F_{m}\right\} \psi(k) .
$$

Here $p_{\mu}=\left(k^{\prime}+k\right)_{\mu}, \quad q_{\mu}=\left(k^{\prime}-k\right)_{\mu}$. 
The wave function of a particle with an arbitrary spin $\psi$ can be written (see, for instance, 31, §31) as

$$
\psi=\frac{1}{\sqrt{2}}\left(\begin{array}{c}
\xi \\
\eta
\end{array}\right) .
$$

Both spinors,

$$
\xi=\left\{\begin{array}{c}
\xi_{\dot{\beta}_{1} \dot{\beta}_{2} \ldots \dot{\beta}_{q}}^{\alpha_{1} \alpha_{2}} \\
{\dot{\dot{\beta}_{p}}}_{\dot{\beta}_{2}}
\end{array}\right\}
$$

and

$$
\eta=\left\{\eta_{\dot{\alpha}_{1} \dot{\alpha}_{2} \ldots \dot{\alpha}_{p}}^{\beta_{1} \beta_{2}}\right\},
$$

are symmetric in the dotted and undotted indices separately, and

$$
p+q=2 s .
$$

For a particle of half-integer spin one can choose

$$
p=s+\frac{1}{2}, \quad q=s-\frac{1}{2} .
$$

In the case of integer spin it is convenient to use

$$
p=q=s .
$$

The spinors $\xi$ and $\eta$ are chosen in such a way that under reflection they transform into each other (to within a phase). For $p \neq q$ they are different objects which belong to different representations of the Lorentz group. For $p=q$, these two spinors coincide. Nevertheless, we will use the same expression (17) for the wave function of any spin, i.e., we will also formally introduce the object $\eta$ for an integer spin, bearing in mind that it is expressed in terms of $\xi$. This will allow us to perform calculations in the same way for the integer and half-integer spins.

In the rest frame both $\xi$ and $\eta$ coincide with a nonrelativistic spinor $\xi_{0}$, which is symmetric in all indices; in this frame there is no difference between dotted and undotted indices. The spinors $\xi$ and $\eta$ are obtained from $\xi_{0}$ through the Lorentz transformation:

$$
\xi=\exp \{\vec{\Sigma} \vec{\phi} / 2\} \xi_{0} ; \quad \eta=\exp \{-\vec{\Sigma} \vec{\phi} / 2\} \xi_{0}
$$


Here the vector $\vec{\phi}$ is directed along the velocity, $\tanh \phi=v$;

$$
\vec{\Sigma}=\sum_{i=1}^{p} \vec{\sigma}_{i}-\sum_{i=p+1}^{p+q} \vec{\sigma}_{i},
$$

and $\vec{\sigma}_{i}$ acts on the $i$ th index of the spinor $\xi_{0}$ as follows:

$$
\vec{\sigma}_{i} \xi_{0}=\left(\vec{\sigma}_{i}\right)_{\alpha_{i} \beta_{i}}\left(\xi_{0}\right)_{\ldots \beta_{i} \ldots}
$$

In the Lorentz transformation (18) for $\xi$, after the operator $\vec{\Sigma}$ has acted on $\xi_{0}$ the first $p$ indices are identified with the upper undotted indices and the next $q$ indices are identified with the lower dotted indices. The inverse situation takes place for $\eta$.

We note that in an external field the components of velocity $\vec{v}$ (and together with them the components of $\vec{\phi}$ ) do not commute, in general. However, to the adopted approximation, linear in the external field, one can ignore this noncommutativity which is itself proportional to the field. Moreover, we are mainly interested in the classical limit of the final result where such commutators are negligible since they are proportional to an extra power of $\hbar$. Therefore, $\vec{v}$ and $\vec{\phi}$ will be treated as ordinary numerical parameters.

Next,

$$
\bar{\psi}=\psi^{\dagger} \gamma_{0}=\psi^{\dagger}\left(\begin{array}{cc}
0 & I \\
I & 0
\end{array}\right) ;
$$

here $I$ is the sum of $2 \times 2$ unit matrices acting on all indices of the spinors $\xi$ and $\eta$. The components of the matrix $\Sigma_{\mu \nu}=-\Sigma_{\nu \mu}$ are:

$$
\begin{gathered}
\Sigma_{0 n}=\left(\begin{array}{cc}
-\Sigma_{n} & 0 \\
0 & \Sigma_{n}
\end{array}\right) ; \\
\Sigma_{m n}=-2 i \epsilon_{m n k}\left(\begin{array}{cc}
s_{k} & 0 \\
0 & s_{k}
\end{array}\right) ; \\
\vec{s}=\frac{1}{2} \sum_{i=1}^{2 s} \vec{\sigma}_{i} .
\end{gathered}
$$

The scalar operators $F_{e, m}$ depend on two invariants, $t=q^{2}$ and $\tau=$ $\left(S^{\mu} q_{\mu}\right)^{2}$. The covariant vector of spin $S_{\mu}$ is defined, e.g., for the state with 
momentum $k_{\mu}$, and is obtained via the Lorentz transformation from the vector of spin $(0, \vec{s})$ in the rest frame:

$$
S^{\mu}=\left(S_{0}, \vec{S}\right), \quad S_{0}=\frac{(\vec{s} \vec{k})}{m}, \quad \vec{S}=\vec{s}+\frac{\vec{k}(\vec{k} \vec{s})}{m(\epsilon+m)} .
$$

In the expansion in the electric multipoles

$$
F_{e}(t, \tau)=\sum_{n=0}^{N_{e}} f_{e, 2 n}(t) \tau^{n}
$$

the highest power $N_{e}$ is obviously $s$ and $s-1 / 2$ for integer and half-integer spin, respectively. In the magnetic multipole expansion

$$
F_{m}(t, \tau)=\sum_{n=0}^{N_{m}} f_{m, 2 n}(t) \tau^{n}
$$

the highest power $N_{m}$ is $s-1$ and $s-1 / 2$ for integer and half-integer spin. The invariant form factors $f_{e, m}(t)$, being Fourier-transformed into the coordinate representation, describe the space distribution of the charge, magnetic moment, and higher multipoles, i.e., describe the finite size of the particle.

Clearly,

$$
f_{e, 0}(0)=1, \quad f_{m, 0}(0)=\frac{g}{2} .
$$

Let us note at last that we have chosen the noncovariant normalization for the amplitude (15), being interested in the Lagrangian referring to the world time $t$ and not to the proper time $\tau$.

\subsection{Effects Linear in the Spin}

As a warm-up exercise and check of our approach, let us reproduce now the well-known result (11) for the case of a constant external field. We start with the terms proportional to $g$-factor. The corresponding term in the scattering amplitude can be written as

$$
\begin{gathered}
\frac{e g}{4 \epsilon} \xi_{0}^{\prime \dagger}\{[\exp \{\vec{\Sigma} \vec{\phi} / 2\}(\vec{s} \vec{B}) \exp \{-\vec{\Sigma} \vec{\phi} / 2\}+\exp \{-\vec{\Sigma} \vec{\phi} / 2\}(\vec{s} \vec{B}) \exp \{\vec{\Sigma} \vec{\phi} / 2\}] \\
+\frac{i}{2}[\exp \{\vec{\Sigma} \vec{\phi} / 2\}(\vec{\Sigma} \vec{E}) \exp \{-\vec{\Sigma} \vec{\phi} / 2\}
\end{gathered}
$$




$$
-\exp \{-\vec{\Sigma} \vec{\phi} / 2\}(\vec{\Sigma} \vec{E}) \exp \{\vec{\Sigma} \vec{\phi} / 2\}]\} \xi_{0}
$$

It is essential that in the considered case of a constant external field, one may put $\vec{k}^{\prime}=\vec{k}, \vec{v}^{\prime}=\vec{v}, \vec{\phi}^{\prime}=\vec{\phi}$, since $\vec{q}=\vec{k}^{\prime}-\vec{k}$ corresponds to the field gradient.

In our further calculations we use the well-known identity

$$
\exp \{\hat{A}\} \hat{B} \exp \{-\hat{A}\}=\hat{B}+\frac{1}{1 !}[\hat{A}, \hat{B}]+\frac{1}{2 !}[\hat{A},[\hat{A}, \hat{B}]]+\ldots,
$$

and the following relationships:

$$
\begin{gathered}
{\left[\Sigma_{i}, \Sigma_{j}\right]=4 i \epsilon_{i j k} s_{k}, \quad\left[\Sigma_{i}, s_{j}\right]=i \epsilon_{i j k} \Sigma_{k}} \\
\cosh \phi=\gamma, \quad \sinh \phi=v \gamma .
\end{gathered}
$$

After simple algebraic transformations, expression (23) reduces to

$$
\frac{e g}{2 m} \vec{s}\left[\vec{B}-\frac{\gamma}{\gamma+1} \vec{v}(\vec{v} \vec{B})-\vec{v} \times \vec{E}\right] .
$$

Let us now discuss the contribution of the convection term

$$
-\frac{e}{2 \epsilon} \bar{\psi}\left(k^{\prime}\right) \psi(k) p^{\mu} A_{\mu} .
$$

We write the product of exponents in the expression

$$
\begin{gathered}
\bar{\psi}\left(k^{\prime}\right) \psi(k) \\
=\frac{1}{2} \xi_{0}^{\prime \dagger}\left[\exp \left\{\vec{\Sigma} \vec{\phi}^{\prime} / 2\right\} \exp \{-\vec{\Sigma} \vec{\phi} / 2\}+\exp \left\{-\vec{\Sigma} \vec{\phi}^{\prime} / 2\right\} \exp \{\vec{\Sigma} \vec{\phi} / 2\}\right] \xi_{0}
\end{gathered}
$$

as

$$
\begin{gathered}
\exp \{\vec{\Sigma} \vec{\phi} / 2\} \exp \{-\vec{\Sigma} \vec{\phi} / 2\} \\
=\prod_{p} \exp \{\vec{\sigma} \vec{\phi} / 2\} \exp \{-\vec{\sigma} \vec{\phi} / 2\} \prod_{q} \exp \{-\vec{\sigma} \vec{\phi} / 2\} \exp \{\vec{\sigma} \vec{\phi} / 2\} .
\end{gathered}
$$

Let us consider a typical factor in this formula:

$$
\begin{aligned}
\exp \left\{\vec{\sigma} \vec{\phi}^{\prime} / 2\right\} & \exp \{-\vec{\sigma} \vec{\phi} / 2\}=\cosh \left(\phi^{\prime} / 2\right) \cosh (\phi / 2)-\left(\vec{n}^{\prime} \vec{n}\right) \sinh \left(\phi^{\prime} / 2\right) \sinh (\phi / 2) \\
+ & \vec{\sigma}\left[\vec{n}^{\prime} \sinh \left(\phi^{\prime} / 2\right) \cosh (\phi / 2)-\vec{n} \cosh \left(\phi^{\prime} / 2\right) \sinh (\phi / 2)\right]
\end{aligned}
$$




$$
-i\left(\vec{\sigma}\left[\vec{n}^{\prime} \times \vec{n}\right]\right) \sinh \left(\phi^{\prime} / 2\right) \sinh (\phi / 2) ;
$$

here $\vec{n}^{\prime}=\vec{v}^{\prime} / v^{\prime}, \vec{n}=\vec{v} / v$. Since we are interested in gradients only as long as they enter together with spin, in the first term, $\cosh \left(\phi^{\prime} / 2\right) \cosh (\phi / 2)-$ $\left(\vec{n}^{\prime} \vec{n}\right) \sinh \left(\phi^{\prime} / 2\right) \sinh (\phi / 2)$, we put $\phi^{\prime}=\phi / 2, \quad \vec{n}^{\prime}=\vec{n}$, after which this term turns to unity. Then, we are discussing the interaction linear in spin, so that the product (29) reduces to

$$
\begin{gathered}
1+\vec{\Sigma}\left[\vec{n}^{\prime} \sinh \left(\phi^{\prime} / 2\right) \cosh (\phi / 2)-\vec{n} \cosh \left(\phi^{\prime} / 2\right) \sinh (\phi / 2)\right] \\
-2 i\left(\vec{s}\left[\vec{n}^{\prime} \times \vec{n}\right]\right) \sinh \left(\phi^{\prime} / 2\right) \sinh (\phi / 2) .
\end{gathered}
$$

When substituted into formula (28), the terms proportional to $\vec{\Sigma}$ cancel out. Now, limiting ourselves to the terms linear in $\vec{q}$, we reduce the spin-dependent part of (27) to

$$
-e \frac{p^{\mu}}{2 \epsilon} \frac{i(\vec{s}[\vec{k} \times \vec{q}])}{m(\epsilon+m)} A_{\mu} .
$$

Let us note further that since $p^{\mu} q_{\mu}=0$, the following identity holds

$$
p^{\mu} q_{\alpha} A_{\mu}=p^{\mu}\left(q_{\alpha} A_{\mu}-q_{\mu} A_{\alpha}\right)=p^{\mu} i F_{\alpha \mu} .
$$

Then, we can put $p_{\mu}=2 m u_{\mu}$, where $u_{\mu}$ is the 4 -velocity. As a result we arrive at the following expression:

$$
-\frac{e}{2 m} \vec{s}\left[2\left(1-\frac{1}{\gamma}\right) \vec{B}-\frac{2 \gamma}{\gamma+1} \vec{v}(\vec{v} \vec{B})-\frac{2 \gamma}{\gamma+1} \vec{v} \times \vec{E}\right] .
$$

The sum of (26) and (32) yields (11). Thus, we have reproduced the wellknown result for the interaction linear in the spin, starting from the relativistic wave equation for an arbitrary spin.

Below we repeatedly use identities of the form (31). In the classical language such a transformation corresponds to discarding in a Lagrangian (or adding to it) a total time derivative. Indeed,

$$
u^{\mu} q_{\mu} \rightarrow u^{\mu} \partial_{\mu}=\gamma\left(\frac{\partial}{\partial t}+\vec{v} \nabla\right)=\gamma \frac{d}{d t} .
$$




\subsection{Effects Quadratic in the Spin}

Let us now investigate the interaction of second order in the spin. The "bare", explicitly quadrupole interaction present in expressions (15) and (16) is

$$
-e \frac{p^{\mu}}{2 \epsilon} f_{e, 2}\left(S^{\alpha} q_{\alpha}\right)^{2} A_{\mu} .
$$

Using the identity (31) and relations (22), and then discarding the total time derivative $\partial / \partial t+\vec{v} \nabla$, we write this interaction as

$$
\begin{aligned}
& L_{2 s}=-e f_{e, 2}\left[(\vec{s} \nabla)-\frac{\gamma}{\gamma+1}(\vec{v} \vec{s})(\vec{v} \nabla)\right] \\
& \times\left[(\vec{s} \vec{E})-\frac{\gamma}{\gamma+1}(\vec{s} \vec{v})(\vec{v} \vec{E})+(\vec{s}[\vec{v} \times \vec{B}])\right] .
\end{aligned}
$$

Using the Maxwell equations and adding a total derivative with respect to $t$, one can show that the tensor $s_{i} s_{j}$ in (34) can be rewritten in the following irreducible form: $s_{i} s_{j} \rightarrow s_{i} s_{j}-(1 / 3) \delta_{i j} \vec{s}^{2}$. Now from the nonrelativistic limit of formula (34), it is clear that this formula describes indeed the interaction with an external field of the quadrupole moment

$$
Q_{i j}=-2 e f_{e, 2}\left(3 s_{i} s_{j}-\delta_{i j} \vec{s}^{2}\right) ; \quad Q=\left.Q_{z z}\right|_{s_{z}=s}=-2 e f_{e, 2} s(2 s-1) .
$$

In the asymptotics, as $\gamma \rightarrow \infty$, the interaction (34) tends to a constant

$$
L_{2 s}=-e f_{e, 2}[(\vec{s} \nabla)-(\vec{v} \vec{s})(\vec{v} \nabla)][(\vec{s} \vec{E})-(\vec{s} \vec{v})(\vec{v} \vec{E})+(\vec{s}[\vec{v} \times \vec{B}])] .
$$

It is well-known that even in the absence of the bare quadrupole term, i.e., at $f_{e, 2}=0$, a quadrupole interaction arises in the nonrelativistic limit due to the convection and magnetic terms in interaction (15). The value of this induced quadrupole moment at an arbitrary spin of the particle is [35] (in the formula below we corrected a misprint in the original paper [35]):

$$
Q_{1}=-e(g-1)\left(\frac{\hbar}{m c}\right)^{2} \begin{cases}s, & \text { integer spin, } \\ s-1 / 2, & \text { half-integer spin }\end{cases}
$$

Here we have explicitly displayed the Planck constant $\hbar$ to show that the induced quadrupole moment $Q_{1}$ vanishes in the classical limit $\hbar \rightarrow 0$, 
$s \rightarrow \infty, \hbar s \rightarrow$ const. Therefore, the interaction of second order in spin proportional to $Q_{1}$ does not influence in fact equations of motion of a classical particle (although it plays a role in atomic spectroscopy [35]).

Still, the convection and magnetic terms in expression (15) induce an interaction of second order in spin which has a classical limit and is therefore of interest for our problem. It is convenient here to start with the convection current interaction. Let us come back to formula (30). Again we put in it

$$
\cosh \left(\phi^{\prime} / 2\right) \cosh (\phi / 2)-\left(\vec{n}^{\prime} \vec{n}\right) \sinh \left(\phi^{\prime} / 2\right) \sinh (\phi / 2)=1 .
$$

In the other terms, linear in $\vec{\sigma}$, we keep only the first power of $\vec{q} \rightarrow-i \hbar \nabla$, in the hope that in the final result (29) $\hbar$ will enter in the combination $\hbar s \rightarrow$ const. Nevertheless, these terms by themselves are small as compared to unity, so that in the classical limit expression (30) can be rewritten as

$$
\begin{gathered}
\exp \left\{\vec{\sigma}\left[\vec{n}^{\prime} \sinh \left(\phi^{\prime} / 2\right) \cosh (\phi / 2)-\vec{n} \cosh \left(\phi^{\prime} / 2\right) \sinh (\phi / 2)\right]\right. \\
\left.-i\left(\vec{\sigma}\left[\vec{n}^{\prime} \times \vec{n}\right]\right) \sinh ^{2}(\phi / 2)\right\} .
\end{gathered}
$$

Clearly, in the product (29) the operators $\vec{\sigma}$ attached to

$$
\vec{n}^{\prime} \sinh \left(\phi^{\prime} / 2\right) \cosh (\phi / 2)-\vec{n} \cosh \left(\phi^{\prime} / 2\right) \sinh (\phi / 2) \text {, }
$$

combine in the resulting exponent into the operator $\vec{\Sigma}$ which vanishes in the classical limit. In this limit only those operators $\vec{\sigma}$ survive that are attached to $\left[\vec{n}^{\prime} \times \vec{n}\right] \sinh ^{2}(\phi / 2)$; they combine into $2 \vec{s}$. Thus, in the classical limit the product (29) reduces, with the account for the second identity (25), to

$$
\exp \left\{\frac{1}{m} \frac{\gamma}{\gamma+1}(\vec{s}[\vec{v} \times \nabla])\right\} .
$$

Let us note that the action of the operator (38) on any function of coordinates, whether it is a vector potential or field strength, amounts to the shift of its argument:

$$
\vec{r} \rightarrow \vec{r}+\frac{1}{m} \frac{\gamma}{\gamma+1} \vec{s} \times \vec{v}
$$

Curiously, just this substitution was pointed out in [20] for the transition from covariant equations linear in spin to noncovariant equations (see (6)). 
Now, taking into account the second term in the expansion of the exponential function (38) and using again the identity (31), we obtain the following expression for the quadratic in spin interaction arising from the convection current:

$$
\begin{aligned}
& -\frac{e}{2 m^{2}} \frac{\gamma}{\gamma+1}(\vec{s}[\vec{v} \times \nabla])\left[\left(1-\frac{1}{\gamma}\right)(\vec{s} \vec{B})\right. \\
& \left.-\frac{\gamma}{\gamma+1}(\vec{s} \vec{v})(\vec{v} \vec{B})-\frac{\gamma}{\gamma+1}(\vec{s}[\vec{v} \times \vec{E}])\right] .
\end{aligned}
$$

Let us discuss now the contribution to the discussed effect due to the magnetic moment. It is convenient to write the term in the scattering amplitude we are interested in (it is proportional to $g$-factor) as

$$
\begin{aligned}
& \frac{e g}{4 \epsilon} \xi_{0}^{\prime \dagger}\left\{\left[\exp \left\{\vec{\Sigma} \vec{\phi}^{\prime} / 2\right\} \exp \{-\vec{\Sigma} \vec{\phi} / 2\} \exp \{\vec{\Sigma} \vec{\phi} / 2\}(\vec{s} \vec{B}) \exp \{-\vec{\Sigma} \vec{\phi} / 2\}\right.\right. \\
& \left.+\exp \left\{-\vec{\Sigma} \vec{\phi}^{\prime} / 2\right\} \exp \{\vec{\Sigma} \vec{\phi} / 2\} \exp \{-\vec{\Sigma} \vec{\phi} / 2\}(\vec{s} \vec{B}) \exp \{\vec{\Sigma} \vec{\phi} / 2\}\right] \\
& +\frac{i}{2}\left[\exp \left\{\vec{\Sigma} \vec{\phi}^{\prime} / 2\right\} \exp \{-\vec{\Sigma} \vec{\phi} / 2\} \exp \{\vec{\Sigma} \vec{\phi} / 2\}(\vec{\Sigma} \vec{E}) \exp \{-\vec{\Sigma} \vec{\phi} / 2\}\right. \\
& -\exp \{-\vec{\Sigma} \vec{\phi} / 2\} \exp \{\vec{\Sigma} \vec{\phi} / 2\} \exp \{-\vec{\Sigma} \vec{\phi} / 2\}(\vec{\Sigma} \vec{E}) \exp \{\vec{\Sigma} \vec{\phi} / 2\}]\} \xi_{0} .
\end{aligned}
$$

Using in this case the first term in the expansion of the exponential function (38), we arrive at the following expression for the contribution proportional to the magnetic moment:

$$
\frac{e g}{2 m^{2}} \frac{\gamma}{\gamma+1}(\vec{s}[\vec{v} \times \nabla])\left[(\vec{s} \vec{B})-\frac{\gamma}{\gamma+1}(\vec{s} \vec{v})(\vec{v} \vec{B})-(\vec{s}[\vec{v} \times \vec{E}])\right] .
$$

The total result for the induced interaction, quadratic in the spin, is

$$
\begin{gathered}
L_{2 s}^{i}=\frac{e}{2 m^{2}} \frac{\gamma}{\gamma+1}(\vec{s}[\vec{v} \times \nabla])\left[\left(g-1+\frac{1}{\gamma}\right)(\vec{s} \vec{B})-(g-1) \frac{\gamma}{\gamma+1}(\vec{s} \vec{v})(\vec{v} \vec{B})\right. \\
\left.-\left(g-\frac{\gamma}{\gamma+1}\right)(\vec{s}[\vec{v} \times \vec{E}])\right]
\end{gathered}
$$

Let us note that in the nonrelativistic limit the induced interaction with magnetic field tends to zero as $v / c$, and that with electric field as $(v / c)^{2}$. 
Moreover, the part of interaction (42) that is not related to $g$-factor, is reducible in spin; in other words, $s_{i} s_{j}$ in it cannot be rewritten as an irreducible tensor $s_{i} s_{j}-(1 / 3) \delta_{i j} \vec{s}^{2}$. Therefore, the interaction (42) is not in fact a quadrupole one. However, its asymptotic behaviour for $\gamma \rightarrow \infty$ is of interest. In this limit

$$
L_{2 s}^{i}=\frac{e}{2 m^{2}}(g-1)(\vec{s}[\vec{v} \times \nabla])[(\vec{s} \vec{B})-(\vec{s} \vec{v})(\vec{v} \vec{B})-(\vec{s}[\vec{v} \times \vec{E}])] .
$$

Surprisingly, the asymptotical formulae (36) and (43) coincide to within a factor and a total time derivative. To prove this, it is convenient to introduce three orthogonal unit vectors

$$
\vec{v} ; \quad \vec{\rho}=\frac{[\vec{v} \times \vec{s}]}{|[\vec{v} \times \vec{s}]|} ; \quad \vec{\zeta}=[\vec{v} \times \vec{\rho}] .
$$

Using the completeness of this basis and the equation $\dot{\vec{E}}=[\nabla \times \vec{B}]$, and discarding a total derivative with respect to $t$, one can check that

$$
\begin{gathered}
{[(\vec{s} \nabla)-(\vec{v} \vec{s})(\vec{v} \nabla)][(\vec{s} \vec{E})-(\vec{s} \vec{v})(\vec{v} \vec{E})+(\vec{s}[\vec{v} \times \vec{B}])]} \\
=[\vec{v} \times \vec{s}]^{2}(\vec{\zeta} \nabla)[(\vec{\zeta} \vec{E})+(\vec{\rho} \vec{B})]
\end{gathered}
$$

coincides indeed with

$$
\begin{aligned}
(\vec{s} & {[\vec{v} \times \nabla])[(\vec{s} \vec{B})-(\vec{s} \vec{v})(\vec{v} \vec{B})-(\vec{s}[\vec{v} \times \vec{E}])] } \\
& =-[\vec{v} \times \vec{s}]^{2}(\vec{\rho} \nabla)[(\vec{\rho}[\vec{v} \times \vec{B}])+(\vec{\rho} \vec{E})] .
\end{aligned}
$$

Thus, there is a special value of the bare quadrupole moment

$$
Q=-2(g-1) \frac{e s^{2}}{m^{2}}, \quad \text { or } f_{e, 2}=(g-1) \frac{1}{2 m^{2}}
$$

(let us recall that we consider now a classical situation, when $s \gg 1$ ), at which the total interaction quadratic in spin, $L_{2 s}+L_{2 s}^{i}$, asymptotically decreases with energy.

The situation resembles that which takes place for the interaction linear in spin. It is well-known (see, for instance, [11, 36, 37]) that there is a special value of $g$-factor, $g=2$, at which the interaction linear in spin decreases 
as $\gamma \rightarrow \infty$. This follows immediately from formula (11) for the first-order Lagrangian. Thus, putting additionally $g=2$, we obtain

$$
Q=-2 \frac{e s^{2}}{m^{2}}, \quad \text { or } f_{e, 2}=\frac{1}{2 m^{2}}
$$

Let us note that the choice $g=2$ for the bare magnetic moment is a necessary (but insufficient) condition of renormalizability in quantum electrodynamics [11, 36, 37]. It is satisfied not only for the electron, but also for the charged vector boson in the renormalizable electroweak theory.

In some respect, however, the situation with the special values (44), (45) of the quadrupole moment differs from the situation with $g$-factor. The conditions (44), (45), as distinct from the condition $g=2$, are not universal, since they are valid only for large spins, $s \gg 1$; in other words, they refer only to classical objects with internal angular momentum. In particular, for the charged vector boson of the renormalizable electroweak theory the bare quadrupole interaction is absent at all, $f_{e, 2}=0$. The quadrupole moment of this particle is (in our language) of the induced nature, it is given by formula (37) at $s=1 \quad g=2$.

\section{A Simple-Minded Aside on the Spin Precession in a Gravitational Field}

In this section we present a simple and general derivation of the equations of spin precession in a gravitational field. This approach not only allows us to easily reproduce and generalize known results for spin effects. Pointed out here remarkable analogy between gravitational and electromagnetic fields allows also to easily transform the results of the previous section to the case of an external gravitational field.

It follows from the angular momentum conservation in flat space-time taken together with the equivalence principle that the 4 -vector of spin $S^{\mu}$ is parallel transported along the particle world-line. The parallel transport of a vector along a geodesic $x^{\mu}(\tau)$ means that its covariant derivative vanishes:

$$
\frac{D S^{\mu}}{D \tau}=0 .
$$


(In this section we restrict our discussion to the effects linear in the spin.) We will use the tetrad formalism natural for the description of spin. In view of relation (46), the equation for the tetrad components of spin $S^{a}=S^{\mu} e_{\mu}^{a}$ is

$$
\frac{D S^{a}}{D \tau}=\frac{d S^{a}}{d \tau}=S^{\mu} e_{\mu ; \nu}^{a} u^{\nu}=\eta^{a b} \gamma_{b c d} u^{d} S^{c} .
$$

Here

$$
\gamma_{a b c}=e_{a \mu ; \nu} e_{b}^{\mu} e_{c}^{\nu}=-\gamma_{b a c}
$$

are the Ricci rotation coefficients [38]. Certainly, the equation for the tetrad 4 -velocity components is exactly the same:

$$
\frac{d u^{a}}{d \tau}=\eta^{a b} \gamma_{b c d} u^{d} u^{c}
$$

The meaning of Eqs. (47), (49) is clear: the tetrad components of both vectors vary in the same way, due to the rotation of the local Lorentz vierbein only.

In exactly the same way, the 4-dimensional spin and velocity of a charged particle with the gyromagnetic ratio $g=2$ precess with the same angular velocity in an external electromagnetic field, by virtue of equation (7) at $g=2$ and the Lorentz equation:

$$
\frac{d S_{a}}{d \tau}=\frac{e}{m} F_{a b} S^{b} ; \quad \frac{d u_{a}}{d \tau}=\frac{e}{m} F_{a b} u^{b}
$$

Thus, the correspondence:

$$
\frac{e}{m} F_{a b} \longleftrightarrow \gamma_{a b c} u^{c}
$$

gets obvious. This correspondence allows one to obtain the precession frequency $\vec{\omega}$ of the 3 -dimensional vector of spin $\vec{s}$ in external gravitational field from expression (10) via the simple substitution

$$
\frac{e}{m} B_{i} \longrightarrow-\frac{1}{2} \epsilon_{i k l} \gamma_{k l c} u^{c} ; \quad \frac{e}{m} E_{i} \longrightarrow \gamma_{0 i c} u^{c} .
$$

This frequency is

$$
\omega_{i}=-\epsilon_{i k l}\left(\frac{1}{2} \gamma_{k l c}+\frac{u^{k}}{u^{0}+1} \gamma_{0 l c}\right) \frac{u^{c}}{u_{w}^{0}} .
$$


The factor $1 / u_{w}^{0}$ in this expression is related to the transition in the left-hand side of Eq. (47) to the differentiation with respect to the world time $t$ :

$$
\frac{d}{d \tau}=\frac{d t}{d \tau} \frac{d}{d t}=u_{w}^{0} \frac{d}{d t}
$$

The quantity $u_{w}^{0}$ is supplied with the subscript $w$ to emphasize that this is a world component of 4 -velocity, but not a tetrad component. All other indices in expression (52) are tetrad ones, $c=0,1,2,3 ; i, k, l=1,2,3$. The corresponding spin-dependent correction to the Lagrangian is

$$
L_{1 s g}=\vec{s} \vec{\omega}
$$

As an illustration of formulae (52), (53), let us apply them to the cases of spin-orbit and spin-spin interactions. We restrict, as is common in the problems discussed, to the linear approximation in the gravitational field. However, in our approach, as distinct from the standard ones, both problems can be easily solved for arbitrary particle velocities.

The tetrads $e_{a \mu}$ are related to the metric as follows:

$$
e_{a \mu} e_{b \nu} \eta^{a b}=g_{\mu \nu}
$$

To linear approximation we can put $g_{\mu \nu}=\eta_{\mu \nu}+h_{\mu \nu}$ and do not distinguish between the tetrad and world indices in $e_{a \mu}$. The ambiguity in the choice of tetrads will be fixed by choosing the symmetric gauge $e_{\mu \nu}=e_{\nu \mu}$. Then

$$
e_{\mu \nu}=\eta_{\mu \nu}+\frac{1}{2} h_{\mu \nu} .
$$

Using expression (48) for the Ricci coefficients, we find to linear approximation

$$
\gamma_{a b c}=\frac{1}{2}\left(h_{b c, a}-h_{a c, b}\right) .
$$

Let us start with the spin-orbit interaction. In the centrally symmetric field created by a mass $M$, the metric is

$$
h_{00}=-\frac{2 k M}{r} ; \quad h_{m n}=-\frac{2 k M}{r} \delta_{m n} .
$$

Here the nonvanishing Ricci coefficients are

$$
\gamma_{i j k}=\frac{k M}{r^{3}}\left(\delta_{j k} r_{i}-\delta_{i k} r_{j}\right), \quad \gamma_{0 i 0}=-\frac{k M}{r^{3}} r_{i} .
$$


Their substitution into formula (52) yields the following expression for the precession frequency:

$$
\vec{\omega}_{l s}=\frac{2 \gamma+1}{\gamma+1} \frac{k M}{r^{3}} \vec{v} \times \vec{r}
$$

In the limit of low velocities, $\gamma \rightarrow 1$, the answer goes over into the classical result [2].

Now we consider the spin-spin interaction. Let the spin of the central body be $\vec{s}_{0}$. Linear in $\vec{s}_{0}$ components of metric, which are responsible for the spin-spin interaction, are:

$$
h_{0 i}=2 k \frac{\left[\vec{s}_{0} \times \vec{r}\right]_{i}}{r^{3}}
$$

Here the nonvanishing Ricci coefficients are

$$
\gamma_{i j 0}=k\left(\nabla_{i} \frac{\left[\vec{s}_{0} \times \vec{r}\right]_{j}}{r^{3}}-\nabla_{j} \frac{\left[\vec{s}_{0} \times \vec{r}\right]_{i}}{r^{3}}\right), \quad \gamma_{0 i j}=-k \nabla_{i} \frac{\left[\vec{s}_{0} \times \vec{r}\right]_{j}}{r^{3}}
$$

The frequency of the spin-spin precession is

$$
\begin{gathered}
\vec{\omega}_{s s}=-k\left(2-\frac{1}{\gamma}\right)\left(\vec{s}_{0} \nabla\right) \nabla \frac{1}{r} \\
+k \frac{\gamma}{\gamma+1}\left[\vec{v}\left(\vec{s}_{0} \nabla\right)-\vec{s}_{0}(\vec{v} \nabla)+\left(\vec{v} \vec{s}_{0}\right) \nabla\right](\vec{v} \nabla) \frac{1}{r} .
\end{gathered}
$$

In the limit of low velocities this formula also goes over into the corresponding classical result [3].

In the conclusion of this section we note that in the case of an external gravitational field there is no covariant expression for the force linear in the particle spin. In other words, the deviation from geodesics of the trajectory of a spinning particle is not described by the Riemann tensor. If it were the case, there would be a unique possible covariant structure, to within a factor (in [6] it equals $-1 / 2 m$ ): $R_{\mu \nu a b} u^{\nu} S^{a b}$. As mentioned already in Section 1, the covariant description (as distinct from our our formulae (52), (53)) contradicts the classical results in the limit of low velocities. 


\section{Equations of Motion of a Spinning Particle in a Gravitational Field}

\subsection{The General Approach}

The equations of motion in an external gravitational field to any order in spin are constructed similarly to the equations of motion in the case of an electromagnetic field.

We start with the elastic scattering amplitude in a weak external gravitational field $h_{\mu \nu}$. We use it as an euristic argument only, and afterwards will go beyond the linear approximation. This amplitude is

$$
-\frac{1}{2} T_{\mu \nu} h^{\mu \nu}
$$

The matrix element $T_{\mu \nu}$ of the energy-momentum tensor between states of momenta $k$ and $k^{\prime}$ can be written as

$$
\begin{gathered}
T_{\mu \nu}=\frac{1}{4 \epsilon} \bar{\psi}\left(k^{\prime}\right)\left\{p_{\mu} p_{\nu} F_{1}+\frac{1}{2}\left(p_{\mu} \Sigma_{\nu \lambda}+p_{\nu} \Sigma_{\mu \lambda}\right) q^{\lambda} F_{2}\right. \\
+\left(\eta_{\mu \nu} q^{2}-q_{\mu} q_{\nu}\right) F_{3} \\
\left.+\left[S_{\mu} S_{\nu} q^{2}-\left(S_{\mu} q_{\nu}+S_{\nu} q_{\mu}\right)(S q)+\eta_{\mu \nu}(S q)^{2}\right] F_{4}\right\} \psi(k) .
\end{gathered}
$$

The scalar operators $F_{i}$ in this expression are also expanded in powers of $\tau=(S q)^{2}$ :

$$
F_{i}(t, \tau)=\sum_{n=0}^{N_{i}} f_{i, 2 n}(t) \tau^{n} .
$$

It can be easily proven that the total number of invariant form factors $f_{i, 2 n}$ is $4 s+2$ and $4 s+1$ for integer and half-integer spin, respectively. The independence of the four tensor structures in (61) is obvious. As for the completeness of the expansion, it can be proven, for instance, by demonstrating that the total number of invariant form factors, as calculated in the annihilation channel, coincides with the above result.

In the generally covariant form, the structure $\left(\eta_{\mu \nu} q^{2}-q_{\mu} q_{\nu}\right) h^{\mu \nu}$ corresponds to the scalar curvature $R$, and $\left[S_{\mu} S_{\nu} q^{2}-\left(S_{\mu} q_{\nu}+S_{\nu} q_{\mu}\right)(S q)+\right.$ $\left.\eta_{\mu \nu}(S q)^{2}\right] h^{\mu \nu}$ corresponds to the product $R_{\mu \nu} S^{\mu} S^{\nu}$, where $R_{\mu \nu}$ is the Ricci 
tensor. Since we are interested in the equations of motion in a sourceless field, the corresponding terms in the expansion (61) will be omitted.

Just as in electrodynamics charge conservation dictates the condition $f_{e, 0}(0)=1$, here energy conservation leads to $f_{1,0}(0)=1$. As to the term in the amplitude (60) which contains $f_{2,0}$, it is convenient to write it in a different form, using the analogy (50) with electromagnetic field. Putting $g=2$ and $(e / m) F_{a b} \rightarrow f_{a b}=\gamma_{a b c} u^{c}$ in the corresponding electromagnetic term

$$
i \frac{e g}{8 \epsilon} \bar{\psi}\left(k^{\prime}\right) \Sigma^{a b} F_{a b} \psi(k),
$$

we arrive at the following contribution to the Lagrangian of the gravitational interaction:

$$
i \frac{1}{4 u_{w}^{0}} \bar{\psi}\left(k^{\prime}\right) \Sigma^{a b} f_{a b} \psi(k)
$$

here, as usual, $u_{w}^{0}=\epsilon / m$. Using for $\gamma_{a b c}$ the linear approximation (54), one can easily see that the expression (62) corresponds indeed to the discussed contribution to the amplitude provided that $f_{2,0}=1$. Thus, in gravity the value of one more form factor at zero momentum transfer $t$ is fixed. It corresponds to conservation of angular momentum. This was pointed out earlier in [39, 40].

Let us now come back to the convection term in formula (60). As in electrodynamics, when we go over here to spinors in the rest frame, the term of first order in spin is written as

$$
-\frac{p^{\mu} p^{\nu}}{8 \epsilon} \frac{1}{m} \frac{u^{0}}{u^{0}+1}(\vec{s}[\vec{v} \times \nabla]) h_{\mu \nu}
$$

Using (31), (54), we obtain

$$
p^{\mu} \nabla_{k} h_{\mu \nu} \rightarrow-p^{\mu}\left(-\partial_{k} h_{\mu \nu}+\partial_{\mu} h_{k \nu}\right) \rightarrow-2 p^{a} \gamma_{a k \nu}
$$

Thus, the expression (63) can be written in terms of the Ricci coefficients:

$$
\frac{1}{u_{w}^{0}} \frac{u^{0}}{u^{0}+1} \epsilon^{m n k} s^{m} v^{n} u^{a} u^{c} \gamma_{a k c}
$$

As can be easily seen, the sum of (62) and (64) reproduces the Lagrangian (53). 


\subsection{Effects Quadratic in the Spin}

Let us investigate now the effects of second order in the spin in the equations of motion in a gravitational field. In the case of a binary star these effects are of the same order of magnitude as the spin-spin interaction when the spins of the components of the system are comparable [28]. The influence of the latter on the characteristics of gravitational radiation becomes noticeable for a system of two extreme black holes [25]. Correspondingly, second-order spin effects in the equations of motion become substantial if at least one component of a binary is close to an extreme black hole [28]. Therefore, the investigation of these effects is not of a purely theoretical interest only. In principle they can be observed with the gravitational wave detectors under construction.

An obvious source of second-order spin effects is the term

$$
L_{2 s g}=-f_{1,2} \frac{1}{8 \epsilon} p^{\mu} p^{\nu}(S q)^{2} h_{\mu \nu}
$$

in the amplitude (60). Due to the relation

$$
\begin{gathered}
p^{\mu} p^{\nu} q_{\alpha} q_{\beta} h_{\mu \nu}=p^{\mu} p^{\nu}\left(q_{\alpha} q_{\beta} h_{\mu \nu}+q_{\mu} q_{\nu} h_{\alpha \beta}-q_{\alpha} q_{\nu} h_{\mu \beta}-q_{\beta} q_{\mu} h_{\nu \alpha}\right) \\
\rightarrow 2 p^{\mu} p^{\nu} R_{\mu \alpha \nu \beta},
\end{gathered}
$$

the Lagrangian (65) can be written in terms of the Riemann tensor:

$$
L_{2 s g}=-\frac{\kappa}{2 \epsilon} u^{a} S^{b} u^{c} S^{d} R_{a b c d}
$$

Instead of $f_{1,2}$, we have introduced a dimensionless parameter $\kappa$ :

$$
f_{1,2}=\frac{\kappa}{2 m^{2}}
$$

Now, it is convenient to use the Petrov representation for the components of the Riemann tesor (see [38]):

$$
\begin{gathered}
E_{k l}=R_{0 k 0 l}, \quad E_{k l}=E_{l k} ; \quad C_{k l}=\frac{1}{4} \epsilon_{k m n} \epsilon_{l r s} R_{m n r s}, \quad C_{k l}=C_{l k} ; \\
B_{k l}=\frac{1}{2} \epsilon_{l r s} R_{0 k r s}, \quad B_{k k}=0 .
\end{gathered}
$$


We limit our discussion to the case of a sourceless gravitational field. Then, at $R_{a b}=0$, further simplifications hold:

$$
C_{k l}=-E_{k l}, \quad B_{k l}=B_{l k}, \quad E_{k k}=C_{k k}=0 .
$$

Finally, we arrive at the following interaction Lagrangian quadratic in the spin:

$$
\begin{aligned}
L_{2 s g}= & -\frac{\kappa}{2 \epsilon}\left[\left(2 \vec{u}^{2}+1\right) E_{k l}-2\left(2-\frac{1}{u_{0}+1}\right) u_{k} u_{m} E_{l m}+\delta_{k l} u_{m} u_{n} E_{m n}\right. \\
& +\frac{1}{\left(u_{0}+1\right)^{2}} u_{k} u_{l} u_{m} u_{n} E_{m n} \\
& \left.-2 u_{0} \epsilon_{k m n} u_{m} B_{n l}+\frac{2}{u_{0}+1} u_{k} u_{m} \epsilon_{l r n} u_{r} B_{m n}\right]\left(s_{k} s_{l}-\frac{1}{3} \delta_{k l} \vec{s}^{2}\right) .
\end{aligned}
$$

To avoid misunderstandings, we note that all three-dimensional indices in this equation (and in (70), (71)) are in fact contravariant.

As in electrodynamics, along with the "bare" interaction (69), there is here an induced interaction quadratic in spin. Its explicit form can be obtained most easily by setting $g=2$ in the electromagnetic formula (42) and by making the substitution (51). We also take into account the correspondence

$$
q_{i} \gamma_{a b c} u^{c}=\left(q_{i} \gamma_{a b c}-q_{c} \gamma_{a b i}\right) u^{c} \rightarrow i\left(\partial_{i} \gamma_{a b c}-\partial_{c} \gamma_{a b i}\right) u^{c} \rightarrow i R_{a b c i} u^{c} .
$$

Finally, using (67) and (68), we obtain the following result for the induced interaction:

$$
\begin{aligned}
L_{2 s g}^{i}= & \frac{1}{2 \epsilon}\left\{\left(2 \vec{u}^{2}-\frac{u^{0}-1}{u^{0}+1}\right) E_{k l}-2\left[2-\frac{1}{u_{0}+1}-\frac{1}{\left(u_{0}+1\right)^{2}}\right] u_{k} u_{m} E_{l m}\right. \\
& +\left[1-\frac{1}{\left(u_{0}+1\right)^{2}}\right] \delta_{k l} u_{m} u_{n} E_{m n}+\frac{1}{\left(u_{0}+1\right)^{2}} u_{k} u_{l} u_{m} u_{n} E_{m n} \\
& \left.-2\left(u_{0}-\frac{1}{u_{0}+1}\right) \epsilon_{k m n} u_{m} B_{n l}+\frac{2}{u_{0}+1} u_{k} u_{m} \epsilon_{l r n} u_{r} B_{m n}\right\} s_{k} s_{l} .
\end{aligned}
$$

As in the electromagnetic case, the induced interaction tends to zero in the nonrelativistic limit $\sim v / c$, and the spin factor in it, $s_{k} s_{l}$, is not an irreducible tensor. 
The asymptotic behaviour of $L_{2 s g}$ and $L_{2 s g}^{i}$ is the same: both Lagrangians increase linearly with energy. However, in this case too the coefficient in the "bare" interaction can be chosen in such a way, $\kappa=1$, that the total Lagrangian of second order in the spin decreases (as well as the analogous interaction in electrodynamics) when the energy tends to infinity. At $\kappa=1$

$$
\begin{aligned}
L_{2 s g}+ & L_{2 s g}^{i}=-\frac{1}{\epsilon\left(u^{0}+1\right)}\left(u^{0} E_{k l}-\frac{1}{u_{0}+1} u_{k} u_{m} E_{l m}\right. \\
& \left.+\frac{1}{2\left(u_{0}+1\right)} \delta_{k l} u_{m} u_{n} E_{m n}+\epsilon_{k m n} u_{m} B_{n l}\right) s_{k} s_{l} .
\end{aligned}
$$

\section{The Gravimagnetic Moment}

There is a profound analogy between the linear in the spin interaction of the magnetic moment with the electromagnetic field

$$
\mathcal{L}_{e m}=-\frac{e g}{4 m} F_{a b} S^{a b}
$$

and the "bare" gravitational Lagrangian (66) which is quadratic in the spin [11]. (Here it is more convenient to write the gravitational Lagrangian, like $\mathcal{L}_{e m}$, for the proper time $\tau$, rather than the world time $t$, i.e., to multiply expression (66) by $\epsilon / m$.) This analogy is based on the following observation [11]. It is well-known that the canonical momentum $i \partial_{\mu}$ enters relativistic wave equations for a particle in electromagnetic and gravitational external fields via the combination

$$
\Pi_{\mu}=i \partial_{\mu}-e A_{\mu}-\frac{1}{2} \Sigma^{a b} \gamma_{a b \mu} .
$$

It follows from the structure of the commutator (or Poisson brackets in the classical limit)

$$
\left[\Pi_{\mu}, \Pi_{\nu}\right]=-i\left(e F_{\mu \nu}-\frac{1}{2} \Sigma^{a b} R_{a b \mu \nu}\right)
$$

that in a sense $-\frac{1}{2} \Sigma^{a b} R_{a b \mu \nu}$ plays the same role in gravity as $e F_{\mu \nu}$ in electromagnetism. It is quite natural then that the gravitational analogue of the electromagnetic spin interaction (72) is

$$
\mathcal{L}_{g m}=\frac{\kappa}{8 m} R_{a b c d} S^{a b} S^{c d} .
$$


One can easily show that expressions (73) and (66) coincide (to within a factor $\epsilon / m)$. It is sufficient to this end to take into account the relation $S^{a b}=\epsilon^{a b c d} S_{c} u_{d}$, as well as the identity

$$
\tilde{R}_{a b c d}=\frac{1}{4} \epsilon_{a b}^{e f} \epsilon_{c d}^{g h} R_{e f g h}=-R_{a b c d}
$$

which is valid for a sourceless gravitational field.

By analogy with the magnetic moment

$$
\frac{e g}{2 m} S^{\mu \nu}
$$

it is natural to define the gravimagnetic moment

$$
-\frac{\kappa}{2 m} S^{a b} S^{c d}
$$

The gravimagnetic ratio $\kappa$, like the gyromagnetic ratio $g$ in electrodynamics, may have in general any value. However, according to our semiclassical arguments, the value $\kappa=1$ in gravity is as preferable as $g=2$ in electrodynamics. In any case, at $g=2$ and $\kappa=1$ the spin equations of motion have the simplest form.

On the other hand, it has been shown in [11] that just this value of the gravimagnetic ratio, $\kappa=1$, follows from the wave equations in the Feynman gauge for the photon and graviton in an external gravitational field. The same value, $\kappa=1$, follows from the squared Rarita-Schwinger equation for $s=3 / 2$ in a gravitational field [11].

A second-order wave equation for an arbitrary spin in a gravitational background had been proposed long ago in [41. For integer spins, its form corresponds also to $\kappa=1$. However, the value of $\kappa$ proposed in [41] for half-integer spins is quite different, it does not tend to unity even in the semiclassical limit $s \rightarrow \infty$. Clearly, such a prescription does not look reasonable: in the semiclassical limit $s \rightarrow \infty$ the parameter $\kappa$ should not change when the spin changes by $1 / 2$.

The situation with spin $1 / 2$ is rather special [11]. The properties of the spin matrices for $s=1 / 2$,

$$
S^{a b}=\frac{i}{4}\left(\gamma^{a} \gamma^{b}-\gamma^{b} \gamma^{a}\right)
$$


are such that the gravimagnetic interaction (73) degenerates here into the mere scalar curvature

$$
\frac{\kappa}{16 m} R
$$

without any consequences whatsoever for the motion of the spin. Obviously, the coefficient at this spin-independent stucture cannot be fixed by the above arguments. Formula (74) would predict for $\kappa=1$ the following wave equation for $s=1 / 2$

$$
\left(-g^{\mu \nu} D_{\mu} D_{\nu}-m^{2}+\frac{1}{8} R\right) \psi=0
$$

(the factor $1 / 2 m$ should be deleted from the expressions (73) and (74) when going over from a Lagrangian in our normalization to a wave equation). Meanwhile, the squared Dirac equation in a gravitational field is

$$
\left(-g^{\mu \nu} D_{\mu} D_{\nu}-m^{2}+\frac{1}{4} R\right) \psi=0
$$

The discrepancy between $(75)$ and $(76)$ can be removed, for instance, by adding

$$
\frac{1}{16 m} R
$$

to the Lagrangian $(73)$ for half-integer spins. This term in no way influences our semiclassical arguments, vanishing in the limit $\hbar \rightarrow 0$. As to the recent proposal 42] to cure the discussed discrepancy by ascribing to the electron (which has no gravimagnetic interaction at all) the gravimagnetic ratio $\kappa=2$, we cannot see in it any real physical meaning.

\section{Multipoles of Black Holes}

But let us come back from elementary particles to macroscopic bodies. For a classical object the values of both parameters $g$ and $\kappa$ depend in general on the various properties of the body. However, for black holes the situation is different. It has been shown in [43] from an analysis of the Kerr-Newman solution that the gyromagnetic ratio of a charged rotating black hole is universal (and equal to that of the electron!): $g=2$.

We will show that for the Kerr black hole the gravimagnetic ratio is $\kappa=1$. This value follows in fact from the analysis of the motion of spin of a black 
hole in an external field done in [24] (though this statement was not explicitly formulated there). We will present here an independent and, in our opinion, simpler derivation of this important result.

At great distance from a Kerr hole, the hole can be considered as a point source of a weak gravitational field. To linear approximation in the field of a hole at rest, the Lagrangian density corresponding to the interaction (66) can be written as

$$
\mathrm{E}=\frac{\kappa}{4 m}(\vec{s} \nabla)^{2} h_{00} \delta(\vec{r}) .
$$

The thus induced correction to the energy-momentum tensor has a single component:

$$
\delta T_{00}=-\frac{\kappa}{2 m}(\vec{s} \nabla)^{2} \delta(\vec{r}) .
$$

In the gauge

$$
\bar{h}^{\mu \nu}{ }_{\nu}=0, \quad \bar{h}_{\mu \nu}=h_{\mu \nu}-\frac{1}{2} \eta_{\mu \nu} h_{\alpha}^{\alpha}
$$

the static Einstein equation for the corresponding correction $h_{00}$ to the 00component of the metric is

$$
\Delta h_{00}=8 \pi k T_{00} .
$$

The correction itself is

$$
h_{00}=\kappa \frac{k}{m}(\vec{s} \nabla)^{2} \frac{1}{r} .
$$

Let us compare $h_{00}$ with the corresponding contribution to the Kerr metric. In the Boyer-Lindquist coordinates this metric is

$$
\begin{gathered}
d s^{2}=\left(1-\frac{r_{g} r}{\Sigma}\right) d t^{2}-\frac{\Sigma}{\Delta} d r^{2}-\Sigma d \theta^{2}-\left(r^{2}+a^{2}+\frac{r_{g} r a^{2}}{\Sigma} \sin ^{2} \theta\right) r^{2} \sin ^{2} \theta \\
+\frac{2 r_{g} r a}{\Sigma} \sin ^{2} \theta d \phi d t,
\end{gathered}
$$

where $\Delta=r^{2}-r_{g} r+a^{2}, \quad \Sigma=r^{2}+a^{2} \cos ^{2} \theta, \quad \vec{a}=\vec{s} / m$. At $r_{g}=0$ the metric (81) describes a flat space in spheroidal coordinates [38]. Meanwhile, it is Cartesian coordinates which correspond in the flat space to the gauge (79). The transition from the spheroidal coordinates to Cartesian ones is carried out with the required accuracy by the substitution

$$
\vec{r} \rightarrow \vec{r}+\frac{\vec{a}(\vec{a} \vec{r})-\vec{r} a^{2}}{2 r^{2}} .
$$


In the Cartesian coordinates the spin-dependent part of the 00-component of the metric

$$
g_{00}=1-\frac{r_{g}}{r}+\frac{r_{g} a^{2}}{2 r^{3}}\left(3 \cos ^{2} \theta-1\right)
$$

obviously coincides with $h_{00}$ from formula (80) at $\kappa=1$. Somewhat more tedious consideration of the space components of the Kerr metric leads to the same result, $\kappa=1$.

Let us note that the motion of the Kerr black hole in an external gravitational field is not described by the Papapetrou equation even if one leaves aside the problem of spin-orbit interaction linear in spin. The point is that this equation refers to the case $\kappa=0$ [14].

It is proven in the same way that for a charged Kerr hole as well the gravimagnetic ratio $\kappa=1$. Moreover, it can be proven that the electric quadrupole moment of a charged Kerr hole also equals

$$
Q=-2 \frac{e s^{2}}{m^{2}}
$$

the value, at which the interaction quadratic in spin decreases with energy. It has been shown [44] that other, higher multipoles of a charged Kerr hole as well possess just those values which guarantee that the interaction of any order in spin (but of course, linear in an external field) asymptotically decreases with increasing energy.

\section{Acknowledgements}

We are grateful to I.A. Koop, R.A. Sen'kov, A.N. Skrinsky, and Yu.M. Shatunov for useful discussions. We are also grateful to D. Bini, G. Gemelli, and R. Ruffini for the advices due to which, hopefully, the present text has become more comprehensible. We greatly appreciate the warm hospitality extended to us by ICRA. The work was supported by the Russian Foundation for Basic Research through Grant No. 98-02-17797 and by the Federal Program Integration-1998 through Project No. 274. 


\section{References}

[1] L.H. Thomas, Nature 117, 514 (1926); Phil. Mag. 3, 1 (1926).

[2] W. de Sitter, Mon. Not. R. Astron. Soc. 77, 155, 181 (1916).

[3] L. Schiff, Phys. Rev. Lett. 4, 435 (1959).

[4] J. Frenkel, Z. Phys. 37, 243 (1926).

[5] V. Bargman, L. Michel, and V. Telegdi, Phys. Rev. Lett. 2, 435 (1959).

[6] A. Papapetrou, Proc. Roy. Soc. London A 209, 248 (1951).

[7] A. Barducci, R. Casalbuoni, and L. Lusanna, Nuovo Cimento A 35, 389 (1976).

[8] F. Ravndal, Phys. Rev. D 21, 2823 (1980).

[9] P.L. Nash, J. Math. Phys. 25, 2104 (1984).

[10] U. Heinz, Phys. Lett. B 144, 228 (1984);

Ann. Phys. (N.Y.) 161, 48 (1985).

[11] I.B. Khriplovich, Zh. Eksp. Teor. Fiz. 96, 385 (1989)

[Sov. Phys. JETP 69, 217 (1989)].

[12] J.W. van Holten, Nucl. Phys. B 356, 3 (1991).

[13] R.H. Rietdijk and J.W. van Holten, Class. Quantum Grav. 9, 575 (1992).

[14] K. Yee and M. Bander, Phys. Rev. D 48, 2797 (1993).

[15] J.P. Costella and B.H.J. McKellar, Int. J. Mod. Phys. A 9, 461 (1994).

[16] M. Chaichian, R. Gonzales Felipe, and D. Louis Martinez, Phys. Lett. A 236, 188 (1997); E-print archive hep-th/9601119.

[17] Ya.I. Azimov and R.M. Ryndin, in Proceedings of the XXXI PNPI Winter School (St. Petersburg, 1997); E-print archive hep-ph/9710433, hep-ph/9707468.

[18] D.Bini, G.Gemelli, and R.Ruffini, in these Proceedings. 
[19] Ya.S. Derbenev and A.M. Kondratenko, Zh. Eksp. Teor. Fiz. 64, 1918 (1973) [Sov. Phys. JETP 37, 968 (1973)].

[20] K. Heinemann, DESY report DESY 96-229; E-print archive physics/9611001.

[21] W. Pauli, Collected Scientific Papers, eds. R. Kronig and V.F. Weisskopf (John Wiley and Sons, 1964), v. 2, p. 544.

[22] H. Batelaan, T.J. Gay, and J.J. Schwendiman, Phys. Rev. Lett. 79, 4517 (1997).

[23] T.O. Niinikoski and R. Rosmanith, Nucl.Instr.Meth. A 225, 460 (1987).

[24] K.S. Thorne and J.B. Hartle, Phys. Rev. D 31, 1815 (1985).

[25] L.E. Kidder, C.M. Will, and A.G. Wiseman, Phys. Rev. D47, R4183 (1993).

[26] L. Blanchet, T. Damour, B.R. Iyer, C.M. Will, and A.G. Wiseman, Phys. Rev. Lett. 74, 3515 (1995).

[27] H.T. Cho, Class. Quantum Grav. 15, 2465, (1998); E-Print archive gr-qc/9703071.

[28] I.B. Khriplovich and A.A. Pomeransky, Phys. Lett. A 216, 7 (1996); E-print archive gr-qc/9602004.

[29] B.M. Barker and R.F. O'Connell, Gen. Rel. Grav. 5, 539 (1974).

[30] A.A. Pomeransky and I.B. Khriplovich, Zh. Eksp. Teor. Fiz. 113, 1537 (1998) [Sov. Phys. JETP 86, 839 (1998)];

E-print archive gr-qc/9710098.

[31] V.B. Berestetskii, E.M. Lifshitz, and L.P. Pitaevskii, Quantum Electrodynamics (Pergamon Press, 1994).

[32] J.D. Bjorken and S.D. Drell, Relativistic Quantum Mechanics, (McGraw-Hill, 1964).

[33] L.L. Foldy and S.A. Wouthuysen, Phys. Rev. 78, 248 (1951). 
[34] I.B. Khriplovich, Parity Nonconservation in Atomic Phenomena (Gordon and Breach, 1991).

[35] I.B. Khriplovich, A.I. Milstein, and R.A. Sen'kov, Phys. Lett. A 221, 370 (1996); Zh. Eksp. Teor. Fiz. 111, 1935 (1997) [Sov. Phys. JETP 84, $1054(1997)]$.

[36] S. Weinberg, in Lectures on Elementary Particles and Quantum Field Theory, eds. S. Deser, M.Grisaru, and H. Pendleton (MIT Press, 1970).

[37] S. Ferrara, M. Porrati, and V.L. Telegdi, Phys. Rev. D 46, 3529 (1992).

[38] L.D. Landau and E.M. Lifshitz, The Classical Theory of Fields (Butterworth-Heinemann, 1975).

[39] I.Yu. Kobzarev and L.B. Okun', Zh. Eksp. Teor. Fiz. 43, 1904 (1962) [Sov. Phys. JETP 16, 1343 (1962)].

[40] F.W. Hehl, A. Macias, E.W. Mielke, and Yu.N. Obukhov, in On Einstein's Path, Festschrift for E. Schucking on the Occasion of His 70th Birthday, ed. A. Harvey (Springer, 1998); E-print archive gr-qc/9706009.

[41] S.M. Christensen and M.J. Duff, Nucl. Phys. B 154, 301 (1979).

[42] R. Aldrovandi, V.C. de Andrade, and J.G. Pereira, E-print archive gr-qc/9804117 v2.

[43] B. Carter, Phys. Rev. 174, 1559 (1968).

[44] A.A. Pomeransky, in these Proceedings. 